\title{
Species abundance and asymmetric interaction strength in ecological networks
}

\author{
Diego P. Vázquez, Carlos J. Melián, Neal M. Williams, Nico Blüthgen, Boris R. Krasnov \\ and Robert Poulin
}

D. P. Vázquez (dvazquez@lab.cricyt.edu.ar), Inst. Argentino de Investigaciones de las Zonas Áridas, Centro Regional de Investigaciones Cientícas y Tecnológicas, CONICET, CC 507, AR-5500 Mendoza, Argentina. - C. J. Melián, National Center for Ecological Analysis and Synthesis Univ. of California, 735 State Street, Suite 300, Santa Barbara, CA 93101, USA. - N. M. Williams, Dept of Biology, Bryn Mawr College, Park Science Building, 101 N. Merion 10 Ave, Bryn Mawr, PA 19010, USA. N. Blüthgen, Lehrstuhl Tierökologie \& Tropenbiologie, Biozentrum, Univ. Würzburg, Am Hubland, DE-97074 Würzburg, Germany. - B. R. Krasnov, Mitrani Dept of Desert Ecology, Jacob Blaustein Inst. for Desert Research, Ben-Gurion Univ. of the Negev, Sede-Boqer Campus, IL-84990 Midreshet Ben-Gurion, Israel and, Ramon Science Center, PO Box 194, IL-80600 Mizpe Ramon, Israel. - R. Poulin, Dept of Zoology, Univ. of Otago, PO Box 56, Dunedin, New Zealand.

\begin{abstract}
The strength of interactions among species in a network tends to be highly asymmetric. We evaluate the hypothesis that this asymmetry results from the distribution of abundance among species, so that species interactions occur randomly among individuals. We used a database on mutualistic and antagonistic bipartite quantitative interaction networks. We show that across all types of networks asymmetry was correlated with abundance, so that rare species were asymmetrically affected by their abundant partners, while pairs of interacting abundant species tended to exhibit more symmetric, reciprocally strong effects. A null model shows that abundance provides a sufficient explanation of the asymmetry structure in some networks, but suggests the role of additional factors in others. Although not universal, our hypothesis holds for a substantial fraction of networks analyzed here, and should be considered as a null model in all studies aimed at evaluating the ecological and evolutionary consequences of species interactions.
\end{abstract}

Ecological interactions have the potential to influence the ecological and evolutionary dynamics of species. However, because usually few interactions within a community are strong and most are weak (Wootton 1997, Bascompte et al. 2006), only a minority of interactions will have the potential to exert such influence. Furthermore, recent studies of interaction networks suggest that interactions tend to be asymmetric, both in terms of "degree" (i.e. number of links per species) in mutualistic and antagonistic binary networks (Bascompte et al. 2003; Vázquez and Aizen 2004, Vázquez et al. 2005b, Guimarães et al. 2006) and in terms of strength in quantitative mutualistic networks (Bascompte et al. 2006). Thus, usually the effect of one species $i$ on another species $j$ does not match the reciprocal effect of $j$ on $i$. These structural characteristics of networks can have profound consequences for the ecological and evolutionary dynamics of interacting species, and suggest that the conceptual models of coevolution based on the assumption of tight reciprocal specialization (Gomulkiewicz et al. 2000, Gandon et al. 2002, Brandt et al. 2005) may not be representative of what happens with the majority of species interactions.

An open question about these apparently pervasive patterns of asymmetry in species interactions concerns the underlying mechanism responsible for them. Recent studies have hypothesized that the asymmetry observed in many interaction networks may result from the distribution of abundance among species (Dupont et al. 2003, Ollerton et al. 2003, Vázquez and Aizen 2004, Vázquez et al. 2005b), but until now there has been no evaluation of this hypothesis in quantitative networks. One possibility is that consumer-resource mutualistic and antagonistic interactions are neutral at 
the individual level, as posited by neutral community theory for competitive interactions (Bell 2000, Hubbell 2001). We hypothesize that if individuals in a community interact randomly, species abundance would determine interspecific interaction frequency and strength and the resulting asymmetry structure; we call this the "abundance-asymmetry hypothesis" (Fig. 1). Under this scenario, abundant species have frequent encounters with individuals of many other species, most of which are relatively rare and specialized given the pervasive rightskewed distribution of abundance (Preston 1962a, 1962b, May 1975). Furthermore, because frequency of interaction can be a good surrogate of interaction strength when there is high variation in interaction frequency (Vázquez et al. 2005a), a species' relative abundance in a community would also determine its ecological effect on another species. Thus, most pairwise interactions in interaction networks are expected to be asymmetric both in terms of degree (specialists interact with generalists) and strength (rare specialists have weak effects on abundant generalists but experience strong effects from them). The hypothesis proposed here is conceptually related to Vermeij's $(1999,2004)$ ideas on the organization of biological systems. Vermeij argues that inequality is a ubiquitous characteristic of interactions among "metabolic entities," and that the prevalent entity, dominant in terms of size, productivity or metabolism, has a greater number of interactions and performs more functions in the system. The crucial consequence of inequality is that prevalent entities and the conditions they create influence disproportionately the global economy of the system. In turn, this structure leads to an increased energy flow among dominant entities.

Here we evaluate the predictions of the abundanceasymmetry hypothesis. We use a database including 43 bipartite networks of both mutualistic (plant-pollinator and plant-ant) and antagonistic (fish-metazoan parasite and mammal-flea) quantitative interaction networks. We develop an original measure of asymmetry, and use a null model representing the abundanceasymmetry hypothesis against which patterns observed in real networks are contrasted.

\section{Material and methods}

\section{Datasets}

We compiled a database on bipartite quantitative interaction networks, including 11 mutualistic (plantpollinator and plant-ant) and 32 antagonistic (fishmetazoan parasite and mammal-flea) quantitative networks (Appendix 1). Data consist of (1) interaction frequency matrices representing quantitative bipartite networks, in which columns correspond to species in one group (e.g. plants or hosts) and rows correspond to species in the other group (e.g. consumers like animal mutualists or parasites) with positive integers in matrix cells representing frequencies of interaction between pairs of species (Appendix 1); and (2) estimates of abundance for each species in the network.
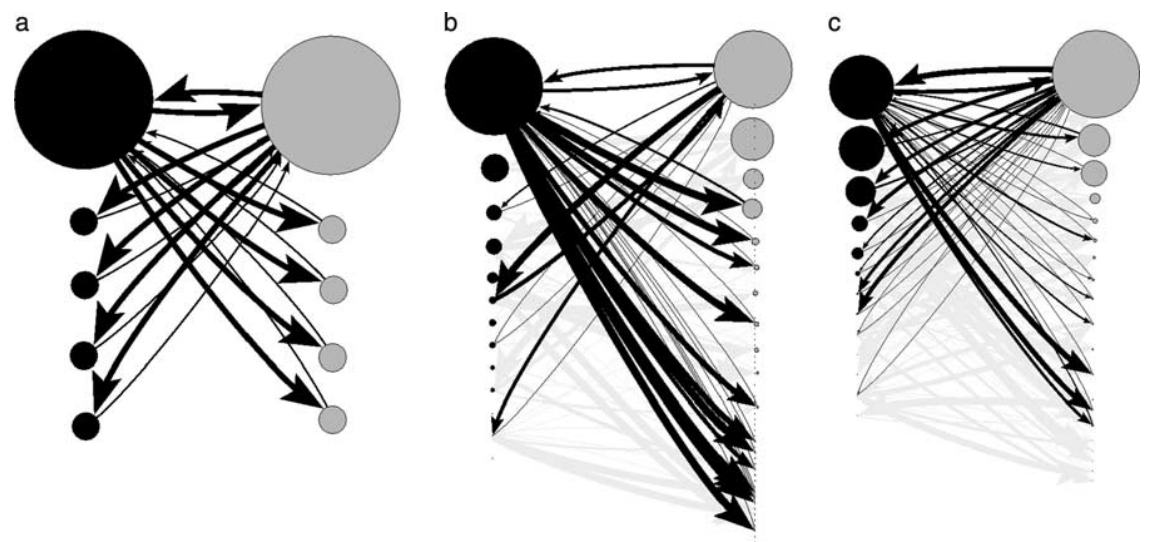

Fig. 1. Graphical representation of quantitative interaction networks to illustrate the abundance-asymmetry hypothesis. Under this hypothesis, abundant species (large circles) are dominant in terms of number of interactions (arrows) and the strength of those interactions for other species (arrow width). Conversely, rare species (small circles) have few interactions and have a minimal effect on abundant species (thin arrows). This model predicts asymmetric effects between rare and abundant species, and symmetric, reciprocally strong effects between abundant species, (a) Simple simulated network; (b) mutualistic network of plants (black circles) and insect pollinators (grey circles) from Llao Llao Municipal Reserve, Bariloche, Argentina (dataset 1, Appendix 1); (c) antagonistic network of mammals (black circles) and fleas (grey circles) from Volovské Vrchy mountains, Slovakia (dataset 39, Appendix 1). For clarity, in (b) and (c) only interactions involving the most abundant species are shown in black; other interactions are shown in light gray. 


\section{Estimation of frequency of interaction and abundance}

Frequency of interaction is defined here as the total number of interactions between hosts and consumers reported in an individual study. In pollination datasets $1-8$, it was estimated as the total number of flowers visited by a pollinator species to each plant species, and in datasets $9-10$ as the number of individuals of a pollinator species collected in sweepnet samples at each plant species. In ant-plant networks, frequency of interaction was estimated as the number of ant individuals of a particular species collected on a plant species. In mammal-flea networks, it was estimated as the mean number of fleas of a given species per individual of a given host species, and in fish-worm networks as the proportion of infected hosts times the abundance of the host (i.e. the number of infrapopulations of the parasite on a particular host species; Vázquez et al. 2005b).

Plant abundance was measured as density of flowering individuals per quadrat sample along transects in pollination studies, and as the number of plant individuals on which any insect activity on nectaries was recorded in plant-ant studies. Except for one dataset (Cold Lake, Appendix 1), host abundance in fish-metazoan networks was estimated as the number of fish collected in the original studies; because in those studies fish were collected for a parasite survey and not specifically to estimate their relative abundance, this figure provides only a rough approximation of species abundance and is likely to underestimate the abundance of abundant species (Vázquez et al. 2005b); the Cold Lake dataset included independent estimates of host abundance, which we used for the analyses. In mammal-flea network datasets, small mammals (orders Insectivora, Rodentia and Lagomorpha) were obtained mainly by trapping, and thus the numbers of each species caught and examined for fleas provide rough estimates of the relative abundance of most host species (Vázquez et al. 2005b).

Except for ant-plant interactions, frequency of interaction with hosts was used as a measure of abundance for consumers. This practice is particularly justified for parasites (fleas and parasitic worms of fish), which spend most of their lives on their hosts. For antplant interactions we had independent abundance estimates for ants, coming from sugar bait censuses (N. Blüthgen, unpubl.).

\section{Quantification of asymmetry}

We define interaction asymmetry as the average mismatch between a focal species' effect on its interaction partners and the reciprocal effect of the interaction partners on the focal species. We assume that the strength of the interaction between two species in two groups of a bipartite network can be defined by two coefficients: $s_{\mathrm{ij}}$, representing the strength of the effect of species $i$ on species $j$, and $s_{\mathrm{ji}}$, representing the strength of the reciprocal effect of $j$ on $i$; these coefficients define two matrices, $\vec{S}=\left|s_{\mathrm{ij}}\right|$ and $\bar{S}=\left|\mathrm{s}_{\mathrm{ji}}\right|$, respectively. Based on previous work suggesting that frequency of interaction is a good surrogate of interaction strength (Vázquez et al. 2005a, Sahli and Conner 2006), we assume that the $\vec{S}$ and $\overleftarrow{S}$ matrices can be derived from a matrix $\mathrm{F}=\left[\mathrm{f}_{\mathrm{ij}}\right]$ describing the frequency of interaction between pairs of species, as also done by others before (Goldwasser and Roughgarden 1993, Bascompte et al. 2006). Particularly, we assume that the effect of a species i on another species $\mathrm{j}$ is proportional to the frequency of interaction between the two species relative to all other interactions of $j$. That is, the strength of the effect of a species $i$ in one group of the bipartite network on a species $j$ in the second group is $s_{i j}=\frac{f_{i j}}{\sum_{m=1}^{I} f_{m j}}$, where I is the total number of species in the first group. Thus, values in the interaction strength matrices $\vec{S}$ and $\bar{S}$ vary between zero (minimal strength) and one (maximal strength). The difference between the elements of the two matrices, $\mathrm{d}_{\mathrm{ij}}=s_{\mathrm{ij}}-s_{\mathrm{ji}}$, is a measure of the symmetry of the strength of each pairwise interaction: a value of zero indicates highly symmetric interaction strength, whereas a value close to 1 or -1 indicates high asymmetry. The sign of the subtraction $\mathrm{d}_{\mathrm{ij}}$ indicates the direction of the asymmetry: positive values indicate that the focal species i exerts a stronger effect on its partner $j$ than the reciprocal effect of $j$ on $i$, whereas a negative value indicates a stronger effect of the partner on the focal species. We define the asymmetry of the interactions of a species $i, A_{i}$, as the average $d_{i j}$ values corresponding to realized interactions $\left(f_{i j}>0\right)$ of $i$; i.e. $A_{i}=\frac{\sum_{j=1}^{J} d_{i j}}{k_{i}}$, where $k_{i}$ is the number of links or "degree" of species i. Thus, a species with an $A_{i}$ value close to 1 would be one that affects strongly its interaction partners but does not experience strong reciprocal effects. Conversely, a species with an $A_{i}$ value close to -1 would be one that experiences strong effects from its interaction partners but that does not exert a strong reciprocal effect. Under the abundanceasymmetry hypothesis we expect a positive correlation between species abundance, $\mathrm{N}_{\mathrm{i}}$, and $\mathrm{A}_{\mathrm{i}}$.

\section{Null model analysis}

Our null model was based on null models of binary networks developed in previous publications (Vázquez and Aizen 2004, Vázquez et al. 2005b). The algorithm 
randomized the total number of individual interactions observed in the original interaction matrix, F. To this end, the algorithm first created a binary matrix, assigning interspecific interactions according to species-specific probabilities, requiring that each species had at least one interaction. As in Vázquez et al. (2005b), the speciesspecific probabilities were proportional to species' relative abundances (probabilities are in fact approximately proportional and not equal to relative abundances because of the requirement that each species receives at least one interaction; this requirement causes probabilities to deviate from relative abundances, especially for rare species). Once the number of filled cells in the original matrix was reached, the remaining interactions were distributed among the filled cells, so that connectance in the original and randomized matrices was the same. The algorithm is written in the Octave/Matlab language, and is available from the first author upon request. Analyses were run under the open source Octave software (http://www.octave.org).

We used meta-analytical techniques to evaluate whether observed correlations between $\mathrm{N}_{i}$ and $A_{i}$ matched those predicted by the null model. If the null model explained the observed relationship between abundance and asymmetry, the difference between observed and expected correlations should not differ significantly from zero. To this end we normalized Spearman's correlation coefficient, r, by applying Fisher's z-transform (inverse hyperbolic tangent), $\mathrm{z}=0.5 \ln$ $[(1+r) /(1-r)]$ (Zar 1999). We then calculated null model deviates from observed normalized correlations, $z_{d}=z_{n}-z_{o}$, where $z_{n}$ and $z_{o}$ are the expected null model and observed normalized correlation coefficients, respectively. To estimate the average deviation between observed and expected correlations across studies, we calculated the weighted mean of $z_{d}$, defined as $\bar{z}_{d}=$ $\Sigma_{\mathrm{w}_{\mathrm{i}}}\left(\mathrm{z}_{\mathrm{d}}\right)_{\mathrm{i}} / \Sigma_{\mathrm{w}_{\mathrm{i}}}$, where the weights are $\mathrm{w}=1 / \operatorname{var}\left(\mathrm{z}_{\mathrm{n}}\right)$ (Rosenthal 1991, Gurevitch et al. 2001). The null hypothesis that observed correlations matched those expected under the null model was rejected if the confidence interval of $\bar{z}_{d}$ did not overlap zero. The confidence interval of $\bar{z}_{d}$ was estimated with the percentile method by bootstrap resampling, defined as the two values that encompass the $100(1-\alpha) \%$ of the distribution of bootstrapped $\bar{z}_{\mathrm{d}}$ values (Manly 1997), with a bootstrap sample size of 10000 .

\section{Results and discussion}

A previous study (Bascompte et al. 2006) of patterns of asymmetry in quantitative plant-pollinator and plantseed disperser networks has shown that most interactions are highly asymmetric. Our results on both mutualistic and antagonistic interactions support this conclusion: there is a high prevalence of asymmetry in networks of all interaction types, suggesting that asymmetric interactions are not just a characteristic of mutualistic interactions, but a more general feature of species interaction networks (Fig. 2). However, our analysis also suggests that asymmetry is not equally prevalent among "hosts" (hosts proper in host-parasite networks and plants in plant-animal mutualistic networks) and "consumers" (parasites or animal mutualists): in most datasets, the latter tend to have more extremely negative asymmetries than the former. That is, hosts tend to have stronger effects on consumers than consumers on hosts. This pattern suggests that bottomup processes have a greater influence than top-down processes in these networks, thus illuminating a
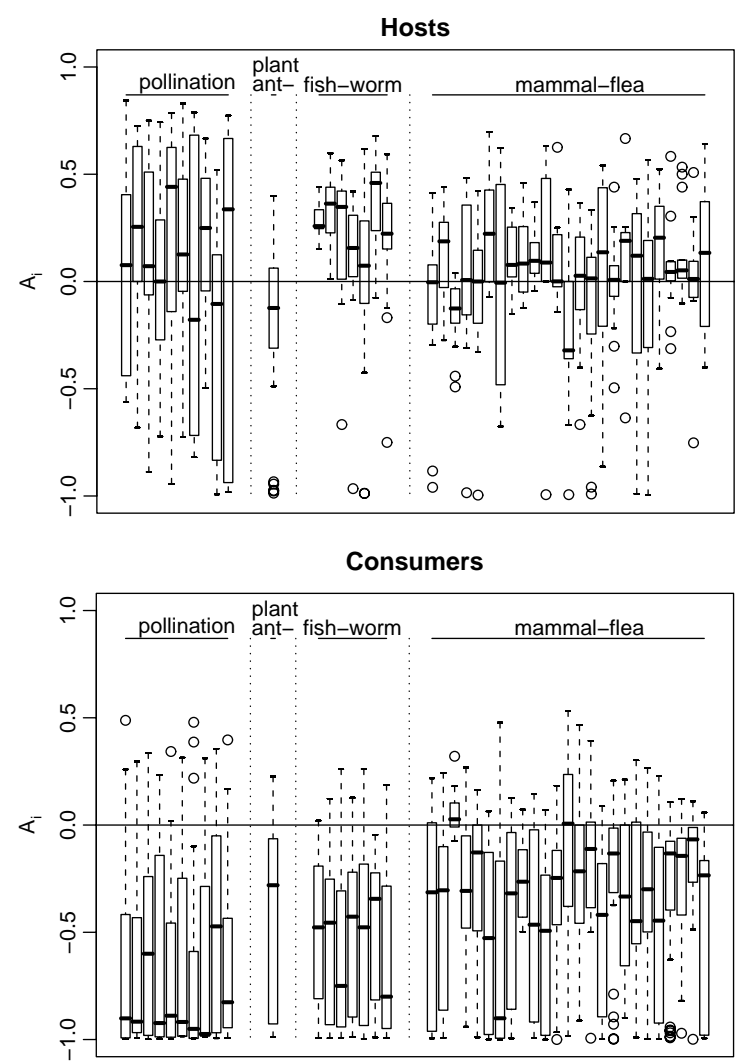

Fig. 2. Box plots showing distribution of asymmetry index, $A_{i}$, calculated for each species in each network. A species with an $A_{i}$ value close to 1 affects strongly its interaction partners but does not experience strong reciprocal effects; a species with an $A_{i}$ value close to -1 experiences strong effects from its interaction partners but that does not exert a strong reciprocal effect. Datasets are ordered from left to right following dataset numbers in Appendix 1. The horizontal line dividing boxes in two indicates the median; box limits are the first and third quartiles of the distribution; whiskers extend to the most extreme data point which is no more than 1.5 times the interquartile range from the box; circles indicate outlying data points falling beyond whisker limits. 
long-standing debate in ecology (Strong 1992, Shurin et al. 2002).

The qualitative prediction of the abundance-asymmetry hypothesis - that there should be a positive correlation between species abundance and their asymmetry index - was supported by the data: with only one exception, there was a generally positive relationship between abundance and asymmetry (Fig. 3, white circles); the exception were the plants in one site in the Nahuel Huapi plant-pollinator dataset (Appendix 1), for which the correlation was negative. Thus, abundant species tend to influence their interaction partners more strongly than their partners influence them; conversely, rare species tend to influence their interaction partners less strongly than the reciprocal effect of their partners on them. These results are consistent with the hypothesis that abundance contributes to generating the asymmetry observed in the mutualistic and antagonistic networks considered here.
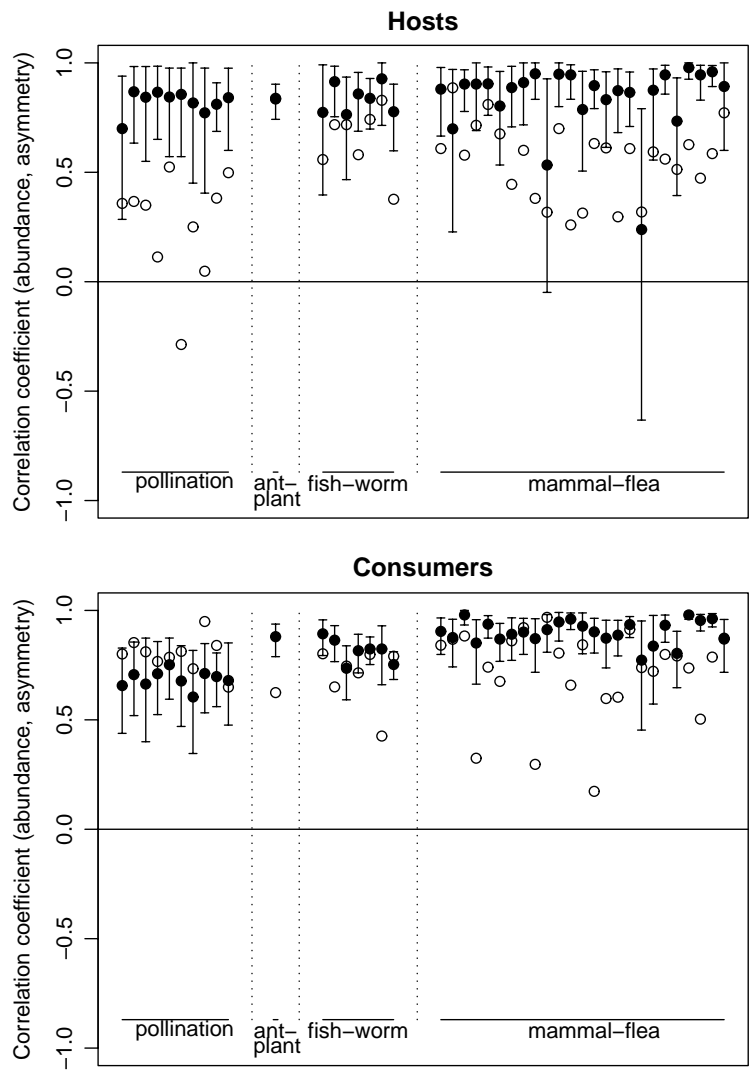

Fig. 3. Correlation between species abundance and the asymmetry index for each interaction type and dataset. White circles are Spearman correlation coefficients calculated for the original data; black circles are Spearman correlation coefficients expected under the null model (mean calculated from 1000 simulated networks); error bars are $95 \%$ percentile confidence intervals (Manly 1997) of null model expectations. Datasets are ordered as in Fig. 2.
Even though abundance contributed substantially to the asymmetric structure observed in these networks, it is important to ask whether abundance alone is a sufficient explanation for such structure, as posited by the abundance-asymmetry hypothesis. We conducted a null model analysis to evaluate this question. Our null model assumed that network structure and the asymmetry resulting from it was entirely the result of a probabilistic process, under which species-specific interaction probabilities were proportional to species relative abundances (Methods). Thus, if the distribution of abundance among species was a sufficient explanation of the asymmetry structure in networks we expected the observed correlation between abundance and the asymmetry index to fall within the range of correlations expected under the null model. This was the case for some datasets, but not for all of them (Fig. 3): for "consumers," observed correlations for 24 of 43 networks fell within the null model expectation, with all but two of the remaining networks falling below the lower confidence limit of the null model; for "hosts," only in 16 of 43 networks did the observed correlation between abundance and the asymmetry index fall within the null model expectation, with most of the remaining networks falling below the lower confidence limit of the null model. Although the mean deviation between null model and observerd normalized correlations was small, it differed significantly from zero (bootstrap confidence limits of $\overline{\mathrm{z}}_{\mathrm{d}}$ : consumers, [0.0006, 0.0069]; resources, [0.0265, 0.0566]), rejecting the null hypothesis that observed correlations matched those expected under the null model. Thus, for a substantial number of datasets, the magnitude of the observed correlation between abundance and asymmetry is lower than that predicted by the null model. The poorer match between observed and expected correlations for "hosts" results mostly from the differential fit of the null model between the two groups in plant-pollinator interactions: for plants, only for one dataset did the observed correlation fall within the null model expectation, with the remaining datasets exhibiting a substantially lower correlation than expected under the null model; conversely, for pollinators the observed correlation matched the null model expectation for eight datasets, with the remaining two datasets exhibiting a greater correlation than expected. The better fit observed for consumers is likely a result of the closer link between abundance and frequency of interaction for this group compared to hosts: whereas abundance was measured independently from frequency of interaction for hosts, both population attributes were by definition the same for consumers (Methods). However, rather than being a methodological artifact, this result reflects a real biological difference between consumers and hosts. 
The results of the null model analysis reported above suggest that in spite of a general effect of species abundance on the asymmetry of interaction strength between interaction partners, abundance alone is not a universal explanation of the distribution of asymmetry. Thus, even though abundance contributes to the asymmetry structure in most datasets, and is a sufficient explanation in a substantial fraction of them, other factors must be invoked as partial explanations of observed patterns in some networks. Phylogenetic constraints and morphological or phenological matching of interacting species have been suggested by others before as potential determinants of the structure of interaction networks (Jordano et al. 2003, Cattin et al. 2004, Stang et al. 2006, Floeter et al. 2007, Santamaría and Rodríguez-Gironés, 2007, Stang et al. 2007), and are certainly a plausible explanation of the above result. Alternatively, however, the mismatch between the observed correlation between abundance and asymmetry and the null model expectation could have resulted from observation uncertainty (Hilborn and Mangel 1997) in the measurement of abundance and interaction frequency. If either or both variables were measured imperfectly (as we know is the case; Methods), this observation uncertainty would lead to a systematic deviation of observed correlations from true correlations, causing the former to be lower than the latter. Only improved measures of abundance and interaction frequency will allow a better understanding of the relative contribution of abundance vs biological traits of species as determinants of the structure of interaction networks.

Because under the abundance-asymmetry hypothesis individuals of abundant species would tend to encounter individuals of other abundant species more often than individuals of rare species, a corollary of the hypothesis is that pairwise interactions among abundant species should exhibit strong reciprocal (symmetric) effects, whereas other pairwise interactions should be more asymmetric. Thus, we expect that the mismatch in strength of pairwise interactions is minimal when the two interacting species are abundant, and that it is greater otherwise. To evaluate this corollary we used the absolute value of the difference of interaction strengths for each pairwise interaction (i.e. the absolute value of the $\mathrm{d}_{\mathrm{ij}}$ subtraction described in Methods for all realized interactions), and calculated its correlation with the product of the relative abundances of interacting species. If the corollary holds, then there should be a negative correlation between absolute differences in strength and the product of abundances, so that reciprocal effects are maximal (i.e. differences in strengths are minimal) when both interacting species are highly abundant. Although generally weak, this correlation was negative for a majority of datasets (Fig. 4; 95\% backtransformed bootstrap confidence of

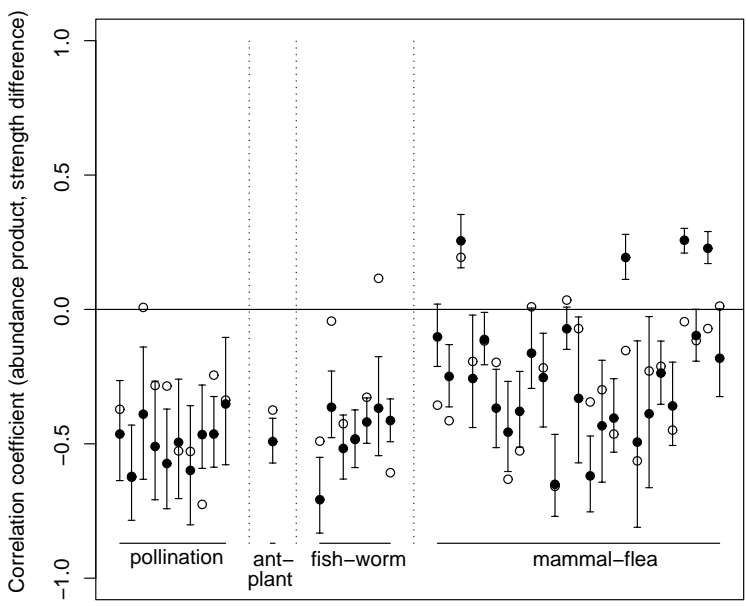

Fig. 4. Correlation between strength difference and abundance product. Spearman correlation coefficients between the absolute difference of the strengths of each pairwise interaction and the product of the abundance of species involved in the interaction. Circle colors and errorbars as in Fig. 3. Datasets are ordered as in Fig. 2.

the correlation coefficient: $[-0.23,-0.40])$. Furthermore, the observed correlation overlaps with the expected correlation under the null model in a substantial number of datasets: the observed correlation fell within null model confidence limits for 21 of 43 datasets, was more strongly negative for nine datasets, and was more positive for the remaining 13 datasets. The mean deviation between null model and observerd normalized correlations was small and did not differ significantly from zero (bootstrap confidence limits of $\left.\overline{\mathrm{z}}_{\mathrm{d}}:[-0.0007,0.0053]\right)$, thus supporting the null hypothesis that observed correlations matched those expected under the null model. However, the fact that the expected correlation under the null model was not always strongly negative suggests the existence of structural network contraints. In particular, connectance (the proportion of non-zero entries in the interaction frequency matrix $\mathrm{F}$ described in Methods) seems to impose an important constraint on the possible values of the correlation between the abundance product and the difference in strength: the expected value of this correlation tends to increase with increasing connectance (Spearman's $r$ between the expected correlation [black circles in Fig. 4] and connectance is 0.53 ). We conclude that the generally negative correlation between the abundance product and the differences in strength, and the relatively good match between the observed correlation and that expected under the null model, support the prediction that the strongest reciprocal effects tend to occur among abundant species.

So far we have discussed our results in merely ecological terms. Are they also relevant for coevolution 
among interacting species? We argue they may be under some circumstances. If the abundance-asymmetry hypothesis holds, and provided that there are differential effects of an interaction on the fitnesses of the genotypes of a focal species, then we expect that such differential effects become stronger with increasing abundance of the species exerting selection (Siepielski and Benkman 2005). Under these circumstances, in which asymmetries in interaction strength would also result in asymmetries in selection among interacting species, our results may have important implications for the coevolutionary dynamics of interacting species. First, unlike most theories of coevolution, the abundance-asymmetry hypothesis implies that specialists will have limited opportunity for coevolution because they are asymmetrically influenced by their abundant, generalized interaction partners. Second, it is the interactions among abundant generalists that have the greatest potential for strong reciprocal effects and, hence, coevolution. Third, geographic variations in the relative abundance of species can lead to a geographic mosaic of coevolution - with pairs of species coevolving in sites where they are both abundant and not coevolving in sites where one or both of them are rare - in line with the Geographic mosaic theory proposed by Thompson (Thompson 1994, 2005, Gomulkiewicz et al. 2000). Although not universal, the abundance-asymmetry hypothesis seems to hold for a substantial fraction of networks analyzed here, with species abundance explaining a large fraction of variation of the observed asymmetry structure. We suggest this hypothesis should be considered as a null model in all studies aimed at evaluating the ecological and evolutionary consequences of species interactions.

Acknowledgements - We thank J. Bascompte, J. Dorado, T. Fukami, W. Langford and J. N. Thompson for discussion and comments on the manuscript. DPV conducted most of this work as a career researcher of CONICET. He was funded initially, and CJM entirely, by postdoctoral fellowships at NCEAS, a Center funded by NSF (Grant DEB-94-21535), the Univ. of California at Santa Barbara, and the Santa Barbara campus. NMW was supported in part by a postdoctoral fellowship from the Smith Fellows program. This is publication no. 560 of the Mitrani Dept of Desert Ecology and no. 227 of the Ramon Science Center.

\section{References}

Bascompte, J. et al. 2003. The nested assembly of plantanimal mutualistic networks. - Proc. Natl Acad. Sci. USA 100: 9383-9387.

Bascompte, J. et al. 2006. Asymmetric coevolutionary networks facilitate biodiversity maintenance. - Science 312: $372-373$.
Bell, G. 2000. The distribution of abundance in neutral communities. - Am. Nat. 155: 606-617.

Brandt, M. et al. 2005. The coevolutionary dynamics of obligate ant social parasite systems-between prudence and antagonism. - Biol. Rev. 80: 251-267.

Cattin, M.-F. et al. 2004. Phylogenetic constraints and adaptation explain food-web structure. - Nature 427: 835-839.

Dupont, Y. L. et al. 2003. Structure of a plant-flower-visitor network in the high-altitude sub-alpine desert of Tenerife, Canary Islands. - Ecography 26: 301-310.

Floeter, S. R. et al. 2007. The macroecology of marine cleaning mutualisms. - J. Anim. Ecol. 75: 105-111.

Gandon, S. et al. 2002. Coevolution between parasite virulence and host life-history traits. - Am. Nat. 160: $374-388$.

Goldwasser, L. and Roughgarden, J. 1993. Construction and analysis of a large Caribbean food web. - Ecology 74: $1216-1233$.

Gomulkiewicz, R. et al. 2000. Hot spots, cold spots, and the geographic mosaic theory of coevolution. - Am. Nat. 156: $156-174$.

Guimarães, P. R. et al. 2006. Asymmetries in specialization in ant-plant mutualistic networks. - Proc. R. Soc. Lond. B 273: $2041-2047$.

Gurevitch, J. et al. 2001. Meta-analysis in ecology. - Adv. Ecol. Res. 32: 199-247.

Hilborn, R. and Mangel, M. 1997. The ecological detective. - Princeton Univ. Press.

Hubbell, S. P. 2001. The unified neutral theory of biodiversity and biogeography. - Princeton Univ. Press.

Jordano, P. et al. 2003. Invariant properties in coevolutionary networks of plant-animal interactions. - Ecol. Lett. 6: 69-81.

Manly, B. F. J. 1997. Randomization, bootstrap, and Monte Carlo methods in biology. - Chapman \& Hall.

May, R. M. 1975. Patterns of species abundance and diversity. - In: Cody, M. L. and Diamond, J. M. (eds), Ecology and evolution of communities. Belknap Press of Harvard Univ. Press, pp. 81-120.

Ollerton, J. et al. 2003. The pollination ecology of an assemblage of grassland asclepiads in South Africa. - Ann. Bot. 92: 807-834.

Preston, F. W. 1962a. The canonical distribution of commonness and rarity: Part I. - Ecology 43: 185-215.

Preston, F. W. 1962b. The canonical distribution of commonness and rarity: Part II. - Ecology 43: 410-432.

Rosenthal, R. 1991. Meta-analysis procedures for social research. - Sage Publications.

Sahli, H. and Conner, J. 2006. Characterizing ecological generalization in plant-pollination systems. - Oecologia 148: 365-372.

Santamaría, L. and Rodríguez-Gironés, M. A. 2007. Linkage rules for plant-pollinator networks: trait complementarity or exploitation barriers? - PLoS Biology 5: e31. doi:10.1371/journal.pbio.0050031.

Shurin, J. B. et al. 2002. A cross-ecosystem comparison of the strength of trophic cascades. - Ecol. Lett. 5: 785-791.

Siepielski, A. M. and Benkman, C. W. 2005. A role for habitat area in the geographic mosaic of coevolution 
between red crossbills and lodgepole pine. - J. Evol. Biol. 18: $1042-1049$.

Stang, M. et al. 2006. Size constraints and flower abundance determine the number of interactions in a plant-flower visitor web. - Oikos 112: 111-121.

Stang, M. 2007. Asymmetric specialization and extinction risk in plant-flower visitor webs: a matter of morphology or abundance? - Oecologia 151: 442-453.

Strong, D. R. 1992. Are trophic cascades all wet? Differentiation and donor-control in speciose ecosystems. - Ecology 73: 747-754.

Thompson, J. N. 1994. The coevolutionary process. - Univ. of Chicago Press.

Thompson, J. N. 2005. The geographic mosaic of coevolution. - Univ. of Chicago Press.

Vermeij, G. J. 1999. Inequality and the directionality of history. - Am. Nat. 153: 243-253.

Appendix 1. Datasets used for the analysis. (available online as Appendix O15828 at www.oikos.ekol.lu.se)
Vermeij, G. J. 2004. Nature: an economic history. - Princeton Univ. Press.

Vázquez, D. P. and Aizen, M. A. 2004. Asymmetric specialization: a pervasive feature of plant-pollinator interactions. - Ecology 85: 1251-1257.

Vázquez, D. P. et al. 2005a. Interaction frequency as a surrogate for the total effect of animal mutualists on plants. - Ecol. Lett. 8: 1088-1094.

Vázquez, D. P. et al. 2005b. Species abundance patterns and the distribution of specialization in host-parasite interaction networks. - J. Anim. Ecol. 74: 946-955.

Wootton, J. T. 1997. Estimates and tests of per capita interaction strength: diet, abundance, and impact of intertidally foraging birds. - Ecol. Monogr. 67: 4564.

Zar, J. H. 1999. Biostatistical analysis. - Prentice Hall. 